\title{
DE L'AMOUR PERDU VERS LES « AMOURS » RETROUVÉS. A PROPOS DES ÉCRITS PRIVÉS ET LITTÉRAIRES DE LA COMTESSE ALEXANDRINE SCHOUVALOFF. ${ }^{1}$
}

\author{
Jitka RADIMSKÁ \\ Université de Bohême du Sud, České Budějovice
}

\begin{abstract}
En): Our study focuses on the private and literary French writings of Princess Alexandrine of Dietrichstein, born Schouvaloff (1775-1847). We firstly mention different approaches to the exploitation of manuscripts by historians and literati and then we briefly describe the life and the particular character of the writings and reading of this francophone noblewoman of Russian origin who from 1797, thanks to her wedding, had lived in the Germanspeaking environment of the Austrian Empire and who, after her divorce in 1804, stayed in Baden near Vienna, in Switzerland or in Italy. We will try to show to which extent the theme of her four unpublished stories on love, Amour allemand, Amour russe, Amour italien and Amour suisse (German love, Russian Love, Italian Love and Swiss Love) can refer to the actual life of this noblewoman originating from Russian nobility, certainly francophone, maybe francophile (this hypothesis is yet to be confirmed) or if these texts were rather inspired and nourished by her pre-romantic reading. The study also comprises the unabridged text of the short story Amour allemand (German Love).
\end{abstract}

Key words (En): Alexandrine of Dietrichstein, born Countess Schouvaloff (1775-1847); private correspondence; literary stories; Amour allemand (unabridged text); Amour russe, Amour italien, Amour suisse ; Pre-Romanticism

Résumé (Fr) : Notre étude s'appuie sur les écrits privés personnels et littéraires, rédigés en français par la princesse Alexandrine de Dietrichstein, née comtesse de Schouvaloff (17751847). ${ }^{2}$ Après avoir mentionné les différentes démarches de l'exploitation des sources manuscrites par les historiens et par les littéraires, nous évoquerons brièvement la vie et le caractère particulier des écrits et des lectures de cette aristocrate francophone d'origine russe ayant vécu à partir de 1797, grâce à son mariage, dans le milieu germanophone de l'empire autrichien et, après son divorce en 1804, ayant séjourné à Baden prés de Vienne, en Suisse où en Italie. Nous essayerons de montrer dans quelle mesure le thème des 4 récits inédits sur l'amour, Amour allemand, Amour russe, Amour italien et Amour suisse, peut se référer à la vie de cette aristocrate issue de la haute noblesse russe, francophone certes, francophile peut-être (hypothèse à confirmer) ou s'il s'agit plutôt de textes inspirés et nourris de ses lectures préromantiques. L'étude comprend le texte intégral du conte Amour allemand.

Mots-clés (Fr) : Alexandrine de Dietrichstein, née comtesse de Schouvaloff (1775-1847) ; correspondance privée ; récits littéraires; Amour allemand (texte intégral), Amour russe, Amour italien, Amour suisse ; le pré-romantisme

\footnotetext{
${ }^{1}$ Il existe deux variantes de son nom de famille utilisées dans les documents officiels archivés : Alexandra Andrewna Schuwalof (l'arbre généalogique) et Alexandrine Andrevna Schouvaloff (la copie française de 1805). Nous préférons la forme utilisée par la comtesse elle-même dans sa correspondance.

${ }^{2}$ Les manuscrits se trouvent dans les archives de la famille Dietrichstein à Brno en Moravie : MZA Brno, fond G 140, boîte 583 (généalogie, chapelle privée, distinction, divorce, souvenirs et mémoires) ; boîtes 584-585 (essais littéraires) ; boîtes 586-587 et 589 (correspondance).
}

Echo des études romanes X/1-2, $2014 \quad$ - 101 -

ISSN: 1804-8358 (Online) 


\title{
Question(s) de méthode(s)
}

\author{
«Aussi est-il plus facile à un texte \\ d'entrer dans le champ littéraire que d'en sortir. »
} Gérard GENETTE

Avec le développement des sciences humaines qui ont bouleversé les conditions du discours tenu sur la littérature, la question de la légitimité de la démarche critique dans l'analyse des textes manuscrits se pose aux chercheurs. Pour les spécialistes de la littérature, les écrits personnels sont objets d'évaluation et de jugement esthétique, les historiens y cherchent avant tout des informations authentiques et prouvées. Selon nous, les débats menés autour de l'exploitation des sources privées manuscrites ont pour motif des questions de méthode : celle de l'analyse et de l'interprétation du fond et de la forme, celle de la présentation des textes en vue de la publication ou celle du statut des sources privées.

Le statut de la source privée (diaire, journal, souvenirs, mémoires, lettres, traité, essai, etc.) peut être éclairé grâce à la théorie de Gérard Genette qui admet l'existence de deux modes : celui de la fiction, qui est de critère essentiellement thématique et de régime constitutif, et celui de la diction, qui est de critère formel ou conditionnel. Le texte de fiction est toujours littéraire (de littérarité constante), par contre le texte de prose non fictionnelle (de littérarité précaire) est littéraire pour qui le juge tel : «ce qui est en cause, c'est la capacité de tout texte dont la fonction originelle, ou originellement dominante, n'était pas d'ordre esthétique », dit-il. (GENETTE, 1991 : 28) Il serait donc souhaitable d'établir une relation de compatibilité entre l'attitude esthétique et l'adhésion pragmatique, bien qu'il soit, d'après lui, "plus facile à un texte d'entrer dans le champ littéraire que d'en sortir». (GENETTE, 1991 : 29) Genette examine les différences narratologiques entre le récit fictionnel et le récit factuel (par ex. autobiographique, biographique, historique) et s'intéresse à la fiction narrative considérée dans son statut pragmatique.

Parmi les critères qui peuvent aider à nous orienter dans nos démarches analytiques, Genette cite la vitesse de la narration : les scènes détaillées, les dialogues rapportés littéralement ou les descriptions étendues qui soulignent la vraisemblance et, par là, communiquent au lecteur une impression de «fictionalisation ». (GENETTE, 1991 : 74) Un autre critère est celui du mode de la narration, concrètement l'accès direct à la subjectivité des personnages. Genette considère comme indices textuels caractéristiques de la fiction narrative les verbes qui expriment les sentiments ou les pensées des personnages, le monologue intérieur ou le style indirect libre. D'après lui «ces tournures subjectivisantes sont incontestablement plus naturelles au récit de fiction, et nous pouvons bien les tenir, fut-ce avec quelques nuances, pour des traits distinctifs de la différence entre les deux types. »(GENETTE, 1991 : 76-77) Le troisième critère est celui de la voix. Dans le cadre de ce critère, le plus important pour distinguer le récit de fiction du récit factuel est la distinction de niveau : "La distinction de niveau est sans doute ici la plus pertinente, car le souci de vraisemblance ou de simplicité détourne généralement le récit factuel d'un recours trop massif aux narrations du second degré. »(GENETTE, 1991: 79) Le caractère factuel est prouvé par la 
relation entre le narrateur et l'auteur de l'histoire : le schèma narrateur $=$ auteur $\neq$ personnage est, selon Genette, typique pour le récit factuel. L'identification de l'auteur avec le narrateur signale que l'auteur même est celui qui relate l'histoire, donc qui se charge de la responsabilité que tout ce qu'il écrit se base sur la vérité. (GENETTE, 1991 : 80) Si la frontière entre réalité et fiction dans les textes d'ordre autobiographique s'efface, cela ne veut pas dire qu'elle n'existe pas. Elle est plutôt floue comme nous le verrons dans la deuxième partie de notre étude.

Les écrits du for privé représentent un volet souvent inexploité, car les essais littéraires et la correspondance sont parfois difficiles à classer. S'y ajoute la problématique d'édition des textes caractérisée dans le passé par l'usage éclectique des documents autographes. Les risques liés à l'interprétation des dossiers autographiques peuvent être éliminés par les approches de la poétique génétique qui peut s'interroger «sur les problématiques les plus diverses: le conditionnement social et psychologique d'un texte en devenir, le rôle des modèles littéraires culturels et idéologiques dans la création littéraire, la part de la volonté et du hasard dans l'évolution du texte, la pression sociale sur l'écrivain, [...] ». (GIFFORD Paul; SCHMID Marion, 2007: 2) Parmi les 4 phases: prérédactionnelle, rédactionnelle, pré-éditoriale, éditoriale, les deux premières nous intéressent particulièrement car les autographes d'Alexandrine Schouvaloff n'ont jamais été édités de son vivant. Dans la première étape de préparation, la phase exploratoire, plus espacée dans le temps et représentée par des listes ou notes de lectures, titres, plans ou scénarios, etc. est suivie par la phase de décision, qui précède réellement la rédaction du texte. C'est l'incipit qui pourra intégrer à la fois la décision, la programmation et le début de la réalisation de l'écrit, voilà pourquoi il méritera notre intérêt. Quant à la phase rédactionnelle qui comprend, elle aussi, les brouillons, les plans plus ou moins développés, les mises au net corrigées ou les notes explicatives, c'est la génétique textuelle qui nous permet de reconstituer chronologiquement le texte et de le rendre lisible. Sa démarche se résume en 4 opérations de recherche : établissement du dossier (critères de l'authenticité et de datation), spécification des pièces (rangement par liasses), classement génétique sur deux axes : paradigmatique (de similarité) et syntagmatique (pour enchaîner les fragments), déchiffrement et transcription (menés sur l'intégralité des pièces manuscrites). La période contemporaine, inspirée par l'anthropologie structurale et la théorie du texte, a permis de retrouver en production littéraire la dimension historique à l'intérieur même de l'écrit : c'est avant tout la critique génétique qui envisage le manuscrit comme objet spécifique, structuré par le temps. "Elle élargit notre vision de la littérature en faisant apparaître, à côté de l'univers du livre et du lecteur, celui de l'écriture et de l'auteur. »(HAY Louis, 2007 : 13) 
Alexandrine et ses amours privés

«Fais l'un pour l'autre par les qualités de nos coeurs, dignes de nous plaire, la différence de pays et d'éducation mettait entre nous un germe de malentendu dont nous ne nous douttions pas nous mêmes. » ${ }^{3}$

Alexandrine Schouvaloff

La princesse Alexandrine de Dietrichstein, née comtesse de Schouvaloff (1775-1847), fille cadette du comte André Pétrovitch Schouvaloff (1744-1789) et de la comtesse Catherine Petrovna, née Soltykoff (1743-1816), naquit à Saint-Pétersbourg. Étant issue du milieu francophone et francophile de la noblesse russe de l'entourage de l'impératrice Elisabeth I ${ }^{\text {ere }}$, puis Catherine II, elle n'est mentionnée dans les ouvrages des historiens tchèques que par rapport à son mari, le prince François Joseph de Dietrichstein (1763-1854), ambassadeur extraordinaire de la cour de Vienne pour les fêtes du couronnement de Paul ${ }^{\text {er }}$. Alexandrine et François Joseph firent connaissance à la cour impériale en Russie en 1796, le mariage eut lieu le 16 juillet 1797 à Pavlovsk, leur fils Joseph naquit en mars 1798 et, en octobre de la même année, les époux Dietrichstein s'installèrent à Vienne. Le mariage ne fut pas heureux. Souffrant d'une maladie de nerfs, elle ne se sentit à l'aise ni dans la famille des Dietrichstein, ${ }^{4}$ ni dans le milieu méprisant des courtisans viennois qui ne comprenaient pas ses manières et dont elle n'arriva pas à supporter l'hostilité et la fausseté. ${ }^{5}$ Son mari se détourna d'elle et obtint le divorce officiel à l'amiable en 1804. Leur fils unique Joseph fut élevé par la famille de son époux. La princesse ne se rendit pas en Russie, son pays natal, elle préféra s'installer avec sa mère Catherine Petrovna en Autriche, à Baden près de Vienne, et faire de longs séjours en Italie. Dès 1805 elle chercha à obtenir des informations sur la parenté entre sa famille natale et celle des Schwaller dont l'une des branches s'était installée en Suisse. ${ }^{6}$ En 1808, elle fut accueillie par le pape à Rome pour préparer sa communion catholique et l'abjuration qui se déroula en 1809. Elle rédigea un écrit sur sa conversion qui ne fut publié qu'en 1879 par sa petite-fille Alexandrine Mensdorff-Pouilly. Elle demanda au pape d'obtenir la permission de construire la chapelle familiale, qu'elle a réussi à obtenir. ${ }^{7}$ En 1824 , elle monta le plan de la fondation d'une maison de charite. ${ }^{8}$ En 1834 elle fut admise par l'impératrice Caroline Auguste de

\footnotetext{
${ }^{3}$ MZA Brno, G 140, boîte 583, inv. 2454, fol. 241.

${ }^{4}$ «Je portais en moi une maladie chronique que j'ignorais. Les émotions du coeur, le changement d'état, ma grossesse, la conduite de votre Pére envers nous me firent une refutation subite et secouerent l'humeur rhumatique que j'avais dans le corps et l'aporterent avec violence à ma tête et sur mes nerfs. (Ce n'est que 9 ans après Lavit en me guerissant de cette humeur m'en a expliqué clairement les effets). » Ibidem.

${ }^{5}$ «Mon coeur avait besoin d'aimer tout ce qui tenait à mon ami. Il les aima avec candeur, avec franchise et crut en être aimée de même. Je dormais en paix, tandis qu'eux fesaient des remarques malignes et ne me pardonnaient pas mon titre d'étrangère. Les usages de Vienne n'étaient pas les miens alors. (Comme ils le sont devenus depuis par l'habitude.) J'étais très enfant quand à l'expériance je disais tout ce qui me passait par l'esprit. » Ibidem.

${ }^{6}$ Cf. MZA Brno, G 140, boîte 583, inv. 2450.

${ }^{7}$ Cf. Les lettres datées de 1815 (Rome) et de 1822 (Fribourg en Suisse). MZA Brno, RAD, boîte 583 , inv. 2451.

${ }^{8}$ MZA Brno, RAD, boîte 585, inv. 2456.
} 
De l'amour perdu vers les « Amours » retrouvés. A propos des écrits privés et littéraires de la comtesse Alexandrine Schouvaloff

Bavière dans l'ordre de la Croix étoilée (Sternkreuzorden), ce qui témoigne qu'elle jouissait d'une grande considération dans les cercles viennois.'

Ayant été éloignée de son fils, Alexandrine réussit néanmoins à éprouver un amour filial, et dans les années à venir à connaître l'âge heureux d'être grandmère. Une vaste correspondance avec son fils Pépi est rédigée en français. Ni classées ni réparties, les lettres sont rangées pêle-mêle dans la boîte mentionnée dans la note 2, pourtant elles permettent de retrouver les dates d'échanges plus vifs au cours de deux périodes : 1814-1821 et 1842-1848. Dans les premières années, le fils s'adresse à sa mère : «Ma chère mère », dans les années 40 il écrit plus spontanément : «Ma chère et bonne maman ». Vu la quantité de ces échanges épistolaires, nous avons choisi trois lettres, rédigées par le «fils éloigné de sa mère » en 1814. Les deux premières, datées du 17 juin et du 14 juillet, nous éclairent sur les préoccupations du jeune comte Joseph (Pépi) à l'âge de seize ans qui y parle de ses études, commente les événements politiques de la guerre contre Napoléon, présente ses soucis et ses espoirs concernant l'avenir de l'Europe. Ses lettres nous révèlent sa personnalité, les relations familiales, le respect filial de Joseph pour son père, son amour pour sa mère.

«Ma trés chère Mère.

Nous venons de voir du grand drame cette heureuse fin qui me remet en état de vous dire les sentiments d'un fils, qui élo[i]gné de vous, ne pouvoit pas même vous écrire, faute de communication. Ainsi François I rend heureux ceux, que Napoléon força de le haïr; en délivrant l'Allemagne, qui l'avoit abandonné. Le plus grand bonheur, oûtre celui de la liberté de ma nation, c'est de vous savoir en bonne santé, de même que notre chére et bonne Grandmaman. Je crois bien lui pouvoir donner à Vienne le climat de la patrie des arts et des sciences, pour vous posséder toutes deux. Daigne le ciel retenir le bras de l'ange destructeur des belles campagnes ô̂ vous vous trouvez; que la paix regne partout, et qu'on rende aux rois, ce qui est aux rois, ainsi qu'on rendit à Dieu ce qui étoit à Dieu. - Il faut bien que je vous donne de mes nouvelles comme aussi de Papa: Papa est à la campagne de Waidlingau non loin de Hütteldorf et jouit de la meilleure santé du monde. Quant à moi, je suis extrémement diligent. Nous avons obtenu l'année passée de Papa la permission que j'aille étudier à l'université, ce dont je ressens une grande joie et ce qui me fait beacoup de bien : l'amour propre y est excité par la diligence de 123 camerades et on y apprend beaucoup et plus qu'on ne s'asquiert en étudiant seul à la maison, on apprend à connoître les hommes, et à s'estimer soi-même, on y voit combien il faut apprécier les vrais amis qu'on a, en voyant combien il est rare d'en trouver. Commencant maintenant le récit de mes études, il m'est trés flatteur de pouvoir le commencer par mon reveil qui se fait à 5 heures et demie chaque jour; (excepté dimanche ồ je me délasse des travaux de la semaine), car à 6 heures et demie vient déja mon répétition de la philosophie ou des mathématiques. Une heure aprés je me rends au collége et j'y reste jusqu'à 10. Je retourne à la maison, passe dans le jardin, ou lis les gazettes. Nous dinons à 1 heure et demi - 2 heures et 3 quarts, alors je reviens au collége, j'y reste jusqu'à 5 heures, je reviens encore au jardin, je goûte et le soir - 9 heures et demie je travaille pour le jour suivant. Les sciences que j'étudie sont le grec, la philosophie, les mathématiques, enfin l'histoire. Ne craignez. pas chére maman qu'à travers tout cela je n'oublie les beaux arts, car je travaille chaque jour 2 heures avec M. Caron pour arranger la belle collection d'estampes de Papa. Ce qui dans cette collection me fait le plus de plaisir, c'est un dess[e] in de la propre main de Sanzio (Raphael) en deux feuilles (quoique déja bien maltraités par le tems) représentant : l'école d'Athènes. M'exercant moimême, je tiens d'achever pour Papa ce mois d'Avril un dess[e]in qui, pour la peine qu'il me coûta à cause de la manière, cherche son égal. C'est une copie d'un tableau de l'histoire de Decius Mus, gravée à l'eau forte, que je m'étois mis en téte de traiter au crayon exactement comme la gravure; j'ai surmonté toutes le [s] difficultés pour me reprimander d'un tel projet, que j'ai appris à ne plus l'exécuter une secondes fois. Maintenant je viens de commencer une copie du : Silenze d'Hannibal

${ }^{9}$ Cf. La lettre de nomination datée du 2 août 1834. MZA Brno, RAD, boîte 583, inv. 2449. 
Carrazzi, que je traiterai au bistre, encouragé par le succés d'une copie que j'ai faite d'une gravure faite d'aprés un tableau d'Angelica Kaufmann: Andromache pleurante la mort d'Hector; et que j'ai traité de la même maniére. J'étudie aussi la langue bohéme. - Cet hiver la rougeole grossoit si fort à Vienne qu'il n'y avoit pas une seule liste de morts, dont je ne puisse assurer qu'elle contenoit du moins 5 personnes, qui en étoient mortes chaque jour. Ici la rougeole est si dangereux que, si vous oseriez de changer de cravatte dans les 10 premiers jours, vous risqueriez de perdre la vie, aussi bien que si vous regardez un moment par la fenêtre dans les 4 premiéres semaines. J'en fus couvert comme de pourpre, et j'eux une fiévre trés forte, pendant laquelle je vis la premiére fois notre ami Theleman, décontenancé, quoiqu'il cherchoit de me le cacher. J'en fus néanmoins guéri si bien, grace à Dieu que Papa me gronde de ce que j'engraisse si fort. Notre bon ami Théleman a eu une maladie trés forte* (*la goutte s'étoit jetée sur sa poitrine), quoique'il ne le croyoit pas, quand on le regardoit, et qu'il sortit toujours, graces à Dieu, il va mieux maintenat; et le médecin l'a condamné à boire de l'eau d'Egra. - Le Baron François de Lagrange est encore à Vienne et dans l'attente d'un petitfils. Il partira bientôt pour Berlin. - George étudie l'économie à Nicolsburg. J'entends que votre maison de Hütteldorf a été vendu de [???] aprés avoir dépensé pour l'arrangement 13000 florins. Chére Maman! portez vous donc bien avec la bonne Grandmaman, que je baise millefois; je vous embrasse tendrement. Theleman présente ses respects. En vous priant de penser quelquefois à moi, j'ai l'honneur d'être, chére Maman, Votre trés-obéissant fils Pepi Dietrichstein. - Cette nuit la ville fut illuminée à cause du retour de notre empereur qui arriva hier matin et entra avec un grand cortège. - Vienne le 17 Juin 1814. ${ }^{10}$

«Trés chére Maman!

M. Debré part aujourd'hui pour trouver le bonheur de vous voir, que je lui envie, c'est encore quelqu'un qui se rend chez vous, et que je ne puis accompagner; recevez donc mille baisers par cette lettre, qui sera plus heureuse que moi, et dont lui s'est chargé de vous la porter. Elle vous verra en bonne santé, comme notre Grandmaman, vous portera nos voeux et vous donnera de nos nouvelles : Papa a été condamné à garder le lit plus de 2 semaines et n'est pas encore retabli tout à fait souffrant d'un rhume comme mr. son fils qui par la même cause a négligé son collége quelques semaines. On a tenu dernièrement un concil sur moi; c'étoit M. Franck, M. Vivenotte et notre médecin Bürchner; le premier s'est trouvé étonné des progrés de ma santé et pria notre ami Theleman de ne rien changer du tout. Celui-ci n'est pas encore tout à fait retabli. George se porte à merveille à la campagne. Notre gros Baon se porte excessivement bien et ira prendre les eaux de Gastein en Salzbourg. - L'heureuse fin des travaux des Allemands encore dignes de leurs ancêtres ne paroit pas devenir l'aurôre d'un âge d'or. Une nouvelle nuit se répand sur la France et surtout sur l'Espagne; et nous craignons pour deux de nos alliés, des inimitiés de leur orgueil. Tel est le fruit de 20 ans de peine!

Adieu chére Maman je vous embrasse avec Grandmaman. Theleman vous présente ses respects. Votre trés obéissant fils Pepi Dietrichstein. - Vienne, le 14 Juillet 1814. ${ }^{11}$

La troisième lettre envoyée de Prague le $1^{\text {er }}$ décembre nous dévoile comment le jeune étudiant Joseph, privé de son précepteur et ami Theleman, se sentit perturbé par la décision sévère de son père et fut laissé dans ses inquiétudes de jeune adolescent. ${ }^{12}$ Il est évident que sa mère lui manquait.

«Ma trés chére mère!

C'est un triste plaisir, que de vous donner des nouvelles de votre fils dans une situation comme la mienne. Eloigné de mes parens, de mes amis, sans nouvelles que les rares et chéries de ma mére, ne sachant pas même le séjour d'un pére que j'aime et que j'ai trahi, aprés avoir appris à connoître les hommes, jeune encore mais pourtant trop tard, ne pouvant écrire une lettre sans savoir qu'elle n'arrivera pas, je prie Dieu de vous faire parvenir celle-ci. Peur-être le ciel a destiné celle ci à avoir le même sort que toutes celles presque que j'ai écrites depuis mon séjour en Bohème. Eh bien, soit,

\footnotetext{
${ }^{10}$ MZA Brno, G 140, boîte 586, inv. 2459, fol. 47-48. Texte intégral.

${ }^{11}$ MZA Brno, G 140, boîte 586, inv. 2459, fol. 50. Texte intégral.

${ }^{12}$ La boîte comprend les lettres (en brouillon et en copie) dans lesquelles le comte de Dietrichstein explique les raisons de cette démarche.
} 
De l'amour perdu vers les « Amours » retrouvés. A propos des écrits privés et littéraires de la comtesse Alexandrine Schouvaloff

que le monde sache, que pour un criminel ce n'est pas une honte de se repentir publiquement. Je vous dirai en peu de mots les malheurs auxquels le ciel m'a destiné. Je reçus un jour, jour fatal, un billet de mon pére, qui me dit, de partir sur le champ pour les frontiéres de la Bohème. Je crûs, que ce n'étoit que pour un parti de plaisir, et à la hate je ne donnois pas même un baiser d'adieu à mon cher ami Theleman. Je lui écrivis de Znaym et d'Iglau, ổ je reçus l'ordre de mon pére, de pénétrer jusqu'à Prague. J'y arrivai le 28 Août et le 29 j'eus une lettre de mon pére, qui me sépara pour jamais de mon ami. Je n'étois point prévenu d'un coup si terrible. Je répondois tendrement à mon pére, mais me mettant à écrire à mon ami, le ressentiment l'emporta, je trahis, je devins criminel. Une cruelle destinée couronne mon malheur: mes lettres furent interceptées par la police et portés chez mon pére par le ministre de la police. Je n'ose pas écrire à mon pére et n'en reçois point de nouvelles. Je ne reçois point de lettre de mon ami, qui est allé se rendre en sa patrie au sein de sa famille. Ma situation est affreuse. Vous seule y pouvez subvenir. Mon pére voyage en Italie, envoyez lui cette lettre si vous savez son séjour. J'ai reçu vos 3 lettres depuis la paix, vous n'avez point reçu mes réponses. La santé de mon pére s'étoit entièrement retablie. J'ai reçu le 29 novembre votre lettre du 22 septembre. Mon oncle Schouvaloff étoit parti pendant mon séjour à Vienne pour Petersbourg. Je ne sais point de nouvelles de ma tante, votre soeur, ni de Mlle sa fille. Adieu ma chére Mére, je vous embrasse tendrement de même que ma chére Grandmaman à qui j'avois aussi écrit. Votre fils Pepi Dietrichstein.

J'embrasse Nastina. - Prague le $1^{\text {er }}$ décembre 1814. $N^{\text {ro }} 3$ depuis la paix. ${ }^{13}$

Dans les années à venir, en tant que mère et grand-mère, Alexandrine Schouvaloff entretint des relations suivies non seulement avec son fils Joseph (Pépi), mais aussi avec sa belle-fille Gabrielle, née Wratislaw de Mitrovitz (1804$1880)$ et ses quatre petites filles Thérèse (1822-1895), Gabrielle (1825-1909), Clothilde (1828-1899) et Alexandrine (1824-1906). Elle alla de temps en temps leur rendre visite en Bohême.

Le thème de l'amour figurant dans le titre de notre étude n'a pas été choisi par hasard. Il peut être pris comme le point de départ du sujet à développer car le manque d'amour, le besoin d'aimer et d'être aimée, au moins nous le supposons, l'ont menée à se convertir et à ouvrir son coeur à l'autrui. Nous avons pu remarquer qu'elle ne pensait pas à elle-même (cf. les dédicaces ou les sous-titres de ses écrits), qu'elle s'intéressait à la postérité de ses proches (enfants ou amis). Parmi les amours retrouvés, la conversion au catholicisme fut, paraît-il, l'un des moments décisifs dans sa vie. Le fait de retrouver la foi chrétienne représenta pour elle une affaire personnelle, intime et privée, comme elle l'avoue en tête de son manuscrit sur la conversion qui se trouve dans les archives de la famille sous forme d'un cahier non paginé ${ }^{14}$ :

«Ce brouillon a été écrit d'après la volonté de mon confesseur, qui désirait qu'il fut publié. Mais je ne puis me résoudre à le faire de mon vivant. »

Alexandrine commence son écrit commémoratif par l'explication des trois motifs guidant sa plume :

«le premier Seigneur est de vous rendre gloire à la face de tout l'univers, le second de me rapeller plus vivement encore tout ce que je vous dois et le troisieme, de laisser à mon fils et à sa posterité un éxemple frappant de vôtre bonté Divine, de la verité incontestable de leur Sainte Religion, et de leur prouver l'obligation absolue qu'ils auront toujours d'y rester fidêles. »

${ }_{13}^{13}$ MZA Brno, G 140, boîte 586, inv. 2459, fol. 51. Texte intégral.

${ }^{14}$ MZA Brno, G 140, boîte 585, inv. 2456, fol. 241. Nous citons d'après cette version manuscrite. 
Puis, elle se souvient de son enfance et du manque d'éducation en matière de la religion dans la famille Schouvaloff :

«Mon Père, hélas! gâté par les écrits de Voltaire ne croyait pas à la Divinité de Jesus Christ et ma mère mariée très jeune et ne connaîssant de la Religion que des pratiques fatigantes et souvent superstitieuses avait embrassé avec ardeur les opinions de mon Père. Tous deux étaient Déïstes. [...] C'est dans ces principes que ma soeur, mes deux frères et moi fumes élevés. Nos gouvernantes et gouverneurs étaient Protestants et ne nous parlaient jamais de Religion. Je ne savais que mon Pater et je le récitais sans même le comprendre. »

Elle récapitule sa vie et les circonstances de sa rencontre avec son futur mari :

«Cependant ma vie n'était pas toujours heureuse. Une mélancholie vâgue s'emparait souvent de mon coeur. Une imagination vive, trop de sensibilité, me fesaient sentir trop vivement la moindre peine. Que l'on ne figure celle que j'éprouvai en perdant à la fois une amie, un cousin cheri et l'Impératrice Catherine qui me comblait de bontés et que la mort venait d'enlever à ses sujets. Pour comble de chagrin la cour se préparant à partir pour Moscou (où devait se faire le courônnement de Paul $\left.1^{e r}\right)$ je devais la suivre avec ma mére et je prévoyais que j'y serai demandée en mariage pour un homme éstimable que je n'aimais pas, mais que je n'avais aucun raison pour refuser. »

Alexandrine se souvient de l'accouchement difficile de son fils et rappelle d'autres moments, jugés malheureux par elle et vécus pendant les années passées à Vienne. Cependant, ne voulant pas écrire l'histoire de sa vie, elle relate celle de sa conversion : une vision en rêve - un suaire porté par deux anges vêtus de blanc le jour de son anniversaire en 1797 ; une lecture révélatrice des quatre Evangiles dans la bible janséniste de Lemaistre de Sacy, offerte à Alexandrine par sa sœur Praskovia Andreevna; elle fit des commentaires sur divers rites en usage dans les communautés religieuses catholique et protestante, etc. ${ }^{15}$ En se rendant compte des différences entre peuples, cultures, usages et habitudes, elle se montra capable de s'inspirer par les idées de l'époque en pleine évolution. La présentation sommaire des écrits intimes de la plume d'Alexandrine Schouwaloff nous a permis de dévoiler non seulement la sincérité de sa foi, mais aussi sa personnalité, sa culture, ses horizons d'attente, ses sentiments. En lisant les extraits de ses textes, nous nous rendons compte de son talent pour exprimer ses émotions, ses idées, ses jugements, voire de son talent d'écrivaine.

\section{Alexandrine, ses lectures et ses écrits littéraires}

« D’une grande naissance, d'une jolie fortune, gâtée par le monde, je voulais être parfaite. Je voulais réunir et les vertus et la science et les talents. J'aprenais avec ardeur et je ne m'apercevais pas que la source de tous les maux, le plus dangereux des vices, la vanité s'emparait de mon coeur et que la vertu la plus chere à Dieu, l'humilité, me manquait entierement. " ${ }^{16}$

Alexandrine Schouvaloff

Le fait qu'Alexandrine s'est mise à écrire des récits littéraires n'est point surprenant, le milieu de la famille Schouvaloff étant favorable à la littérature. Son

\footnotetext{
${ }^{15}$ Le fait de se convertir au catholicisme n'a pas été exceptionnel à cette époque-là. Cf. La typologie des conversions des femmes par NiQUEUX Michel (2009).

${ }^{16}$ Conversion. MZA Brno, G 140, boîte 585, inv. 2456, fol. 241.
} 
De l'amour perdu vers les « Amours » retrouvés. A propos des écrits privés et littéraires de la comtesse Alexandrine Schouvaloff

père, le comte André Petrovitch, libre-penseur, correspondant de Voltaire et poète francophone, fut auteur de l'Épître à Voltaire (1765), de l'Épître à Ninon de l'Enclos (1774) et de l'ode À Voltaire (1779) et d'autres œuvres en vers. Il fut le préfacier des traductions de certains auteurs français en russe, membre d'une Commission pour la rédaction des ouvrages d'histoire et auteur d'une chronologie de l'histoire russe (1787). Sa sœur Praskovia Andreevna, mariée Golitsyn (17671828), fut traductrice et auteure de deux romans en langue française : Mélise (1813) et Lettres du Duc de P*** (1816). S'ajoutent les rencontres d'Alexandrine, contemporaine de François René de Chateaubriand (1768-1848), avec les milieux et les cultures différents de l'Europe de la première moitié du XIX ${ }^{\mathrm{e}}$ siècle, ses compétences de lectrice avertie, l'inspiration trouvée dans le vécu de sa vie privée et, rappelons-le, son talent littéraire. Pour prolonger nos recherches concernant ses inspirations littéraires, il s'avère utile de nous renseigner plus en détail sur les livres de sa bibliothèque particulière qui faisait parti de la bibliothèque recomposée de plusieurs fonds particuliers dans les années $50 \mathrm{du} 20^{\text {e }}$ siècle et déposée au château à Úsov en Moravie du Nord. ${ }^{17}$ La collection Dietrichstein, installée par les beaux-parents d'Alexandrine Karl Johann de DitrichsteinProskau-Leslie (1728-1808) et Marie Christine, née de Thun (1738-1788) à Lipník nad Bečvou (Leipnik), propriété des Dietrichstein depuis la bataille de la Montagne Blanche, fut enrichie par les générations suivantes. Parmi les livres qui y sont conservés, une Bible russe de la fin du $18^{\mathrm{e}}$ siècle rappelle, parmi d'autres, les liens des Dietrichstein avec la famille Schouvaloff. (MAŠEK Petr, 1990 : 81) Pour le moment, nous n'avons à la disposition qu'une liste de nouveautés en lectures, rédigée par Alexandrine elle-même. Le manuscrit intitulé «Nouveautés en lectures ${ }^{18}$ mentionne 20 ouvrages parus autour de 1831. Le corpus des nouveautés comprend des sujets actuels, contemporains et variés qui dépassent largement le goût des lectrices de la bibliothèque bleue.

«La Contemporaine en Egypte» [pour faire suite aux souvenirs d'une femme, sur les principaux personnages de la République, du Consulat de l'Empire et de la Restauration, par Ida Saint-Elme (=Maria Johanna Elselina Verfelt) $]^{19}$

«Les Intimes par Michel Raimond [auteur français de Macon]

"L'écume des mers [ou la sorcière des eaux, roman d'aventures adapté de l'anglais] par Cooper»

"Une femme à 40 ans - Mlle Lemerslier» [Marguerite Louise Viberti Lemercier, romancière italienne]

"L'homme de la nature et l'homme policé par Paul de Kock»

\footnotetext{
${ }^{17}$ De nos jours, la collection se trouve réinstallée au château à Vranov nad Dyjí en Moravie du Sud.

${ }^{18}$ MZA Brno, RA Dietrichstein, boîte 584, inv. 2455, 1f inséré dans le cahier.

${ }^{19}$ Ida Saint-Elme, dite « la Contemporaine », de son vrai nom Maria Johanna Elselina Verfelt, mais aussi connue sous les nom de Elselina Vanayl de Yongh ou Elzelina Tolstoy van Aylde-Jonghe (1776-1845) est une aventurière, courtisane et écrivaine néerlandaise. Fille d'un pasteur, elle se maria en 1792, mais quitta les Pays-Bas après la conquête française pour suivre ses amants dans leurs campagnes militaires. Courant ainsi les champs de bataille napoléoniens, elle devint la maitresse de nombreux officiers français, comme le général Moreau dont elle fut la presqu'épouse, ou le maréchal Ney. À partir de 1801, elle se lança sans succès dans la carrière d'actrice, et voyagea en Italie, en Égypte, en Allemagne et en Russie. Elle connut la célébrité en publiant en 1827 ses souvenirs sous le titre de Mémoires d'une Contemporaine, qui firent scandale et connurent un prodigieux succès. Ils lui valurent son surnom de «Casanova femelle » ou bien de «Veuve de la Grande Armée », mais ils furent soupçonnés d'avoir été arrangés par quelques hommes de lettres. http://fr.wikipedia.org/wiki/Ida_Saint-Elme [14.5.2014]
} 
De l'amour perdu vers les « Amours » retrouvés. A propos des écrits privés et littéraires de la comtesse Alexandrine Schouvaloff

« Le Chiffonnier par M. Signol » [Alphonse Signol et Stanislas Macaire]

«Le Duc et le page par Lamothe Langon» [roman de mœurs, histoire romancée de l'exil de Charles X]

"Le bonnet vert par Mery» [Joseph]

«La Prima Donna et le boucher » [Clément Burat de Gurgy, Edmond Burat de Gurgy]

«Le Roi des Ribauds par Jacob» [(=Paul Lacroix) ; histoire du temps de Louis XII $]^{20}$

«Une Tuerie de Cosaques par God. Cavaignac » [scènes d'invasion, roman] ${ }^{21}$

«Barnave par Jules Janin » [1831 $]^{22}$

«La peau de chagrin» par Balzac.

"La croix du meurtre - Elza Voiart» [dernier roman d'August Lafontaine, traduction libre par Mme Élise Voïart (=Anne-Élisabeth-Élise Petitpain, Mme Jacques-Philippe)]

«Contes misanthropiques par Henri Berthoud» [Samuel Henry Berthoud, Charles Lemesle]

"Proverbes dramatiques par Cavaignac»

"Memoires et souvenirs du comte Lavallette » [Antoine Marie Chamans ; publiées par sa famille et sur ses manuscrits]

"Revue de Paris 22 et 23 es volumes"

«Etudes et discours historiques par Chateaubriand» [sur la chute de l'Empire romain, la naissance et les progrès du christianisme et l'invasion des barbares]

« Notre Dame de Paris » [par Victor Hugo]

À condition qu'il s'agisse des livres qui ont été lus par la princesse elle-même, ce document peut témoigner de ses lectures non seulement du point de vue de la réception littéraire, mais aussi du point de vue de l'inspiration pour porter plus loin son écriture et sa création littéraire.

Les écrits essais littéraires d'Alexandrine Schouvaloff sont assez variés du point de vue du genre : deux journaux intimes (des années 1797-1798 et 1804), un brouillon du Plan de mes mémoires, des souvenirs (Mes souvenirs. Pour mes enfants et mes amis. Renseignements sur ma famille), un texte commémoratif relatant sa conversion au catholicisme (édition posthume par sa petite-fille), conte satirique allégorique avec le chat comme protagoniste et narrateur (Mémoires de Romina Grobis $^{23}$ ), biographie romancée du personnage historique de Louis de Bourbon Grand Condé, treize pièces de théâtre (4 tragédies et 9 comédies dont la seule achevée s'intitule Une matinée à Venise), plusieurs traités de civilité (Du but

\footnotetext{
${ }^{20}$ Paul Lacroix, plus connu sous les pseudonymes de P. L. Jacob ou du Bibliophile Jacob (18061884) est un polygraphe érudit français. Il composa d'un côté des romans et de l'autre des livres d'histoire, puis il mêla les deux genres dans plusieurs publications. Ses premiers romans eurent du succès et firent école, malgré les difficultés de lecture qu'offrait l'imitation du vieux langage. Ses romans historiques, souvent réimprimés et traduits en plusieurs langues, contribuèrent pour une grande part à propager le goût du Moyen-Âge, qui se répandit alors en France et en Europe jusque dans les arts. Ses publications bibliographiques eurent la même influence sur le goût des livres : il continua à cet égard la mission de Charles Nodier. http://fr.wikipedia.org/wiki/Paul_Lacroix [14.5.2014]

${ }^{21}$ Jacques Louis Éléonore Godefroy Cavaignac (1800-1845), nouvelliste et journaliste républicain, principal opposant à la Monarchie de Juillet. http://fr.wikipedia.org/wiki/Godefroi_Cavaignac\#Publications [14.5.2014]

${ }_{22}$ Gabriel-Jules Janin (1804-1874), journaliste et romancier. Il fut parmi les fondateurs de la Revue de Paris et du Journal des Enfants. Il se fait connaître en 1827 avec le roman L'Âne mort et la femme guillotinée. La Confession en 1830, un peu moins profond, mais au style encore plus remarquable, et Barnave en 1831, où il attaque la famille d'Orléans. Entre-temps, il entre comme critique au Journal des Débats où il reste quarante ans. Son autorité le fait surnommer «le prince des critiques». http://fr.wikipedia.org/wiki/Jules_Janin [14.5.2014]

${ }^{23}$ Le texte intégral du conte a été publié par Martina MusiLOvá (2014 : 132-138).
} 
de l'homme, De la Religion, Des Vertus, Des manieres, De l'Instruction, Des Talents, De la Toilette, De la Santé), des écrits moralistes (Mon Testament moral au faveur de mon fils et des jeunes gens qui voudront en faire leur profit, aux Demoiselles de toutes les classes, A mes petites filles), contes populaires du milieu champêtre, récits d'amour comprenant 4 contes ou nouvelles (Amour allemand, Amour italien, Amour russe, Amour suisse, ${ }^{24}$ ) et 2 romans par lettres (Amour anglais, Amour autrichien), quelques poèmes rarissimes, etc. ${ }^{25}$

\section{Alexandrine et ses amours retrouvés}

«Élargir notre vision de la littérature en faisant apparaître, à côté de l'univers du livre et du lecteur, celui de l'écriture et de l'auteur. »

Louis HAY

Pour découvrir ses stratégies d'écriture, nous allons dans cette étude brièvement présenter et comparer à travers les incipits les 4 contes intitulés par la princesse elle-même Amour. Les quatre milieux définis par les épithètes allemand, italien, russe, suisse n'ont pas été choisis comme cadre spatial de ses histoires d'amour, ils correspondent aux quatre contrées dont Alexandrine ne fit la connaissance personnelle que de trois, en y séjournant. La poétique des quatre espaces présente des caractéristiques différentes qui d'après nous reflètent son vécu réel, la possibilité de de communiquer de façon littéraire ou active dans une langue (français, italien), la curiosité pour l'histoire des origines de sa nation (folklore russe), le retour à travers la lecture à l'époque chevaleresque (le gothique littéraire). Ce qui les relient comme fil d'Ariane, c'est l'accent porté vers le sentiment religieux, le besoin d'un être humain d'aimer et d'être aimé, de croire, de rêver.

Le thème de l'amour entre homme et femme n'est pas dominant dans tous les contes - parfois c'est la vengeance qui organise tout le récit et opère le comportement des protagonistes. C'est le cas de l'Amour allemand 26, récit inachevé et moins travaillé que les trois autres, qui n'est qu'une esquisse : l'intrigue schématique (avec le motif du philtre magique), temps de l'histoire peu défini (l'époque des templiers), lieu peu précisé (château Adelsberg), personnages - clichés (le comte et la comtesse d'Adelberg, leur fils Ernest et Ida, sa fiancée et nièce du comte, Conrad, frère de la comtesse, et Alstophe avide de vengeance, son écuyer Ruperd et la sorcière Herda). Ces indices et l'inspiration conventionnelle due à la méconnaissance du milieu germanophone nous suggèrent l'hypothèse qu'il s'agit du premier conte du cycle des récits d'amour, nourri de ses lectures. Les reprises et les corrections des formulations dans le manuscrit témoignent pourtant du souci de l'auteure de styliser les propos au mieux. Si le discours

\footnotetext{
${ }^{24}$ Ce conte n'est pas mentionné par CERMAN (2011 : 397).

${ }^{25}$ Les écrits inédits d'Alexandrine Schouwaloff ont été présentés et en partie analysés dans les travaux de Martina MusilovÁ (2011 : 1-83) et Martina Musilová (2014 : 63-112).

${ }^{26}$ MZA Brno, G 140, boîte 584, inv. 2455, cahier colorié avec un cœur en papier collé au centre de la couverture, fol. 1-16. Voir le texte intégral ci-dessous.
} 
indirect de la narration manque de vivacité, deux dialogues plus animés par l'émotion des locuteurs dévoilent et dénoncent le secret de leurs passions et témoignent du talent de l'auteure et de l'art de s'exprimer de vive voix : il s'agit du dilemme entre l'amour spontané d'Ida et son obéïssance en vue de la famille d'une part, et le motif conduisant Astolphe à l'idée de faire mourir ceux qu'il considère responsables de son destin malheureux.

L'histoire du conte Amour russe ${ }^{27}$, située dans le pays natal de l'auteure, est beaucoup plus travaillée et retravaillée et existe en deux versions manuscrites. Le récit fut animé par les descriptions des lieux et des fêtes folkloriques en Russie ancienne, et enrichi par des commentaires explicatifs qui s'adressaient au public étranger pour lequel la contrée russe, avec tous ses rites et ses habitudes, représenta un pays méconnu et exotique. Igor assiste sans être vu à la séance magique de jeunes filles animée par la protagoniste Tatiana et l'auteure trouve indispensable de décrire et de commenter ce jeu (comme les autres qui animent le cadre spatial et idéologique du conte) dans le passage paratextuel au bout de la page et à la fin du texte :

"- Mes amis, dit-elle en s'adressant à ses compagnes, il est tems d'apprendre que nous sachions quel sera sera notre sort. - Aux doux accents de cette voix, Igor crut qu'un ange avait parlé. » [...]

"Ces jeux sont encore en usage en Russie. Voyés la notterie à la fin de cette nouvelle. Notte 1 : Jeux que l'on fait depuis la veïlle de Noël jusqu'au jour des Rois reste sans doutte du paganisme ainsi qu'il est aisé de le voir par les noms de quelques anciennes divinités qui se trouvent dans les chansons que l'on chante pendant ces jeux et que le peuple prononce, croyant dire des paroles qui n'ont aucun sens. »

Une correction (parmi maintes autres, rappelons-le) dans l'incipit du conte en question concerne les différences entre les rites de la foi orthodoxe grecque et catholique. Les reprises corrigées font partie de la stratégie d'adapter l'histoire à des horizons d'attente des croyants chrétiens catholiques et de rendre plus compréhensibles toutes les circonstances de la première rencontre des protagonistes :

"C'était la veïlle des Rois Noel. Le Boyard Alexis rentrait dans son Palais tenant par la main le jeune et bel Igor, fils de son ami le plus intime et l'espérence de la Russie. Viens! lui dit-il, viens voir ma Tatiana. Elle brille comme le rubis elle est fraiche comme la rose, et si tu l'aimes elle est à toi. Viens! l'obscurité nous favorise cachés deriere une porte grillée, nous la verons sans en être vus. Le coeur d'Igor battait avec force violance pendant le discours du vieillard. Il savait que Tatiana fille d'Alexis, était la plus belle des filles de Moscou et que le Boyard Yaroslaw son Pere ta tui destinait au mariage desirait vivement les voir amis par les liens de mariage. Igor agé de vingt trois ans n'avait jamais aimé. Quel trouble ne dut-il éprouver dans cet instant!"

Le dénouement moral de l'Amour russe a été retravaillé (prolongé et restylisé) dans la version copiée pour souligner l'apport bénéficiaire de la christianisation de la Russie. Le peuple glorifie son souverain qui, après avoir vu le sacrifice de deux êtres qui s'aimaient tendrement, respecte l'éclat de la divinité et renonce à son mariage prévu avec Tatiana :

\footnotetext{
${ }^{27}$ La version de la plume de la comtesse. MZA Brno, G 140, boîte 584, inv. 2455, cahier de 22 ff. Il existe une copie de ce récit comprenant des épreuves et des notes de l'auteure : MZA Brno, G 140, boîte 584 , inv. 2455 , cahier au ruban paginé $1-86+6$ pp. de notes (= ff. 469-560).
} 
«O Dieu Puissant et juste! [...] Sois béni mille fois de nous avoir donné en Tsat qui sait imiter ta clémence et qui,en récompensnat la valeur et a vertu prépar ainsi le bonheur de la Russie. [...] Bientôt les événements qui ont eu lieu dans ces souterrains circulent de bouche en bouche. La joie éclate de toute part, les noms d'Igor, de Sophie, de Tatiana sont associés à celui du Tsar, mille acclamations apprennent au souverain que le peuple approuve son choix et au'il se réjouit de son bonheur. »

Le cadre temporel des débuts du christianisme est remplacé dans le conte Amour suisse par celui des débuts des Temps Modernes, cependant le registre qui mélange et associe l'amour entre homme et femme à l'amour divin garde les mêmes proportions. Le couple amoureux d'Anaïs et d'Émile qui sont séparés par deux branches religieuses différentes, le protestantisme et le catholicisme, cherchent leur consolation dans la belle nature des montagnes et des vallées jurassiennes et les conseils chez un vieux prêtre. L'incipit de l'Amour suisse ${ }^{28}$ montre bien la progression de l'écrivaine dans les descriptions poétiques qui égalent les peintures de la prose poétique d'un Chateaubriand.

«Les premiers rayons de soleil doraient déjà les sommets du Jura. Les rochers du Valois éclattants de lumière laissaient voir leurs éguilles à travers des vapeurs légéres et mobiles qui tantôt s'élevaient en nuages et tantôt retombaient enrosées dans les sombres forêts de sapins dont les collines sont couvertes. Le vieux Sigfried appuyé sur sa fenêtre contemplait ce spectacle avec ravissement. Tous les jours depuis plus de vingt ans il venait en joü̈r à la même heure. C'était celle de la priere, ensens pur que son coeur offrait avec délice à son Créateur. La cloche de Thonon annoncait l'Angelus du matin. Siegfrid la tête baissée, les mains jointes se revenuïlle et dit : Seigneur O mon Dieu, bénissés, bénissés ses enfants, ses troupeaux et des champs. Mon Dieu! Sainte Vierge! C'est vous qui m'avaient ramené dans le sein de l'Eglise. Faite, o Mere de Misericorde, que je ne trahisse jamais ma sainte croyance, que ma famille y soit toujours fidelle, que nous aimions Dieu de l'amour des Anges a present et dans l'éternité et que nous devenions saints à force de l'adorer. Un profond silence succède à ses paroles. Le veillard est absorbé dans sa contemplation intérieure mais un leger bruit d'une porte qui s'entromra l'en retire et lui fait tourner les yeux vers la porte d'une chambre dont le battant à peine entrouvert laisse voir le joli minois de sa petitte fille Laurette. "Mon pere, dit-elle, j'apporte le déjeuner. »

Si nous le comparons à l'incipit de l'Amour allemand, nous voyons la différence non seulement dans la langueur et le style de la description :

"L'Horloge avait sonné minuit. Les moines du couvent de Saint Ada(e)lberg prosternés dans les voûtes de l'Eglise, offraient en silence leur tribut à l'Eternel. Instant sublime où l'âme pure dégagée de ses liens terrestres s'unit intimement à son créateur, instant consolateur où l'âme plus faible lui offre ses peines et lui demande le repos. O! Qui peut calculer l'encens d'amour et de vertus qui s'élève vers Dieu dans ces sacrés silences.

Un étranger se trouvait dans l'Eglise. Jeune encore, beau, malheureux, envelopé d'un manteau blanc des Templiers, il était à genoux près d'une chapelle écartée. La faible lueur de la lampe [fol. 2v] du maître autel venait frapper l'image de la Vierge qui se trouvait devant lui.

O! Sainte Mère de Dieu, lui disait-il, vous voyés mon sacrifice. Donnés moi des forces pour le supporter. »

L'incipit ne se borne pas à la présentation du cadre spatial d'un récit, du milieu exotique ou pittoresque, il introduit en même temps à la scène les personnagesclés, principaux ou secondaires du point de vue de leur rôle dans l'histoire relatée

${ }^{28}$ MZA Brno, G 140, boîte 584, inv. 2455, 36 ff. (= ff. 131-167). 
et porteurs du message idéologique de l'auteure : un étranger sans nom /le templier Conrad/ revenant dans son pays des croisades (Amour allemand), le boyard Alexis avec le jeune et bel Igor qui s'attend à voir en cachette Tatiana, la plus belle fille de Moscou qu'il croit aimer (Amour russe), le vieux Siegfrid plongé en pleine contemplation de la nature jurassienne au lever du jour et priant (Amour suisse), le jeune noble vénitien Ottavio Vanaldi qui reste envoûté et touché par la voix fraîche et douce qui fesait palpiter son coeur /celle de Céleste/ (Amour italien).

«La lueur éclairait ta lagune. Les à demi voilée laissait tomber quelques rayons sur la lagune argentée les le Canal de la Giudeca et de la lagune argentée s'élevaient les clochers de Venise qui se se dessinaient en masses noires sur l'azur foncé du ciel. Une seule gondole se balançait doucement sur les ondes. Deux voix célestes en sortaient et le gondolier debout appuyé sur la rame semblait enchanté captivé par le doux charme de ces doux sons.Vanaldi était envelopé de son manteau et debout sur le bord runge de la rive des ésclavous avait les yeux fixés sur la barque légere. Dieu! se disait-il à lui-même. Que de charmes doit renfermer ce petit point noir qui se dessine sur cette nappe brillante. Ces sons qui ravissent âme mes sens, ne peuvent appartenir qu'tax [???]les à l'âme la plus pure, aux traîts les plus touchants. Une des deux voix plus fraiche et plus douce que l'autre était celle qui fesait palpiter son coeur. Ottavio Vanaldi avait vingt quatre ans. »

Cependant les différences bien nuancées des débuts de ces quatre récits résultent de la stratégie générique de la narration prise par l'auteure qui renvoie son lecteur vers la lecture et l'interprétation prévues.

\section{Le texte intégral de l'Amour allemand}

[fol. $2 \mathrm{r}]$ « Amour allemand. ${ }^{29}$

L'Horloge avait sonné minuit. Les moines du couvent de Saint Ada(e)lberg prosternés dans les voûtes de l'Eglise, offraient en silence leur tribut à l'Eternel. Instant sublime où l'âme pure dégagée de ses liens terrestres s'unit intimement à son créateur, instant consolateur où l'âme plus faible lui offre ses peines et lui demande le repos. O! Qui peut calculer l'encens d'amour et de vertus qui s'élève vers Dieu dans ces sacrés silences.

Un étranger se trouvait dans l'Eglise. Jeune encore, beau, malheureux, envelopé d'un manteau blanc des Templiers, il était à genoux près d'une chapelle écartée. La faible lueur de la lampe Ifol. 2v] du maître autel venait frapper l'image de la Vierge qui se trouvait devant lui.

O! Sainte Mère de Dieu, lui disait-il, vous voyés mon sacrifice. Donnés moi des forces pour le supporter.

Hors du cloître la lune de à moitié voilée faisait glisser un faible rayon sur les vitraux de l'Eglise et sur les athées qui croissaient aux pieds des murs. La paix de la nuit n'était interrompue que par le bruit douteux d'une marche incertaine. La vieille Herda érrait parmi les broussailles. Son regard sinistre cherchait l'herbe dont le charme devait [quelques mots illisibles biffés] détruire la paix de l'innocence et la livrer au crime. La sorcière déséchée [fol. 3r] écartait les athées et sa bouche infaste pronocait à voix basse et sepulcrale des paroles inconnues. Deux fois elle avait fait le tour du couvent et allait commencer le troisième. Mon main-bras l'arrête. Qui es-tu s'écria-telle? - Paix Herda! C'est Ruperd. Ah! Ah! reprit Herda avec une grimace horrible. J'eusse été étonnée de rencontrer ici autre chose qu'un vilain corbeau comme toi,-prends garde cependant Ruperd. Est-ce ton Demon Ruperd qui t'envire pour interrompre mon opération magique et pour la rendre ma peine inutile? Vas-t-on! Au diable! Ruperd, va et chercher toi même l'herbe qu'il faut à ton maître, pour lui, je ne m'en mêla plus. Maudite sorcière, s'écria Ruperd veux-tu que mon maître m'afferme. Et que m'importe, répondit la vieille. Je resterai [fol. 3v] bien aprés de mauvais

\footnotetext{
${ }^{29}$ Pour plus d'authenticité, nous respectons le texte qui reflète l'usage de l'époque et nous gardons les retouches provenant de la main de son auteure (cf. la photographie à la page 120).
} 
De l'amour perdu vers les « Amours » retrouvés. A propos des écrits privés et littéraires de la comtesse Alexandrine Schouvaloff

sujets sans toi dans ce monde. - Vois cette piece d'or Herda - elle est à toi si tu peux me donner cette herbe. Quand tu m'en offrirais dix mille, Ruperd, il n'est plus en ma puissance de la trouver a présent. En disant ces mots elle s'éloignait en boitant. Ruperd l'arrêta. Ecoute, Herda, écoute ou je te tue à l'instant même. Choisis ou de la mort ou de cette bourse. Astolphe sait que tu cherches cette herbe, il t'attend avec impatience. Je suis venu ici de sa part, s'il apprend que j'ai interrompu ton travail, que j'ai rompu le silence que tu nous avais recommandé, sa fureur n'aura de bornes. Arange, dis ce que tu voudrais mais ou ce fer ou cet or doivent me reprendre de la discretion. En disant ces paroles il tenait sa main droite armée d'un poignard [fol. 4r] cachée sur la poitrine [mot illisible] et de la gauche il lui montrait une bourse remplie de monnaie. Herda fera sur lui un regard sombre et grinceant les dents elle partit d'un éclat de rire convulsif. Donne la bourse, lui dit-elle, et prends ceci. Dis au Seigneur Astolphe que la liqueur contenue dans ce flacon vaut mieux que toutes les herbes du monde et quand il parvient à en faire avaler sept gouttes à la Demoiselle d'Adelsberg, elle ne revera plus qu'à lui. Au revoir, Ruperd, au revoir à ton lit de mort. Ne me suis pas, tu ne peux plus m'atteindre, déja la lune va descendre éclaire le cimetiere, déja le corbeau a vôlé trois fois sur la tombe à revenir dans trois jours Ruperd. Ruperd furieux s'élancea vers elle Herda. Elle avait disparu, en vain chercha-t-il la trappe qui avait pu la dérober à sa vue. Des épines sans nombres entouraient [fol. 4v] la place oùla-d'où la sorciere lui avait parlé dit son terrible adieu. Saisi luimême d'une terreur involentaire, il demeura longtems immobile et reprit ensuite lentement le chemin étroit et raide du chateau.

Le repos n'avait pas encore aproché de ses murs. On y atendLe Seigneur d'Adelsberg y était attendu avec impatience. Il revenait de la terre Sainte et depuis trois jeurs la Comtesse nuits son épouse avait veïllé pour l'attendre. Assise dans la salle des Chevaliers, elle tenait [mots illisibles biffés] la courone nuptiale qui devait orner le front de la jeune Ida au jour de son mariage et se plaisait à orner de pierres precieuses et les branches du romarin dont elle était composée. Ida, nièce de son époux, filait [fol. 5r] en silence auprés d'elle. Une douce satisfaction, une vive impatience se paignaient dans les yeux de la Comtesse. Une timide mélancholie était imprimée sur le front d'Ida et la Comtesse souriait en pensant que l'absence d'Ernest, son fils, en était seule la cause. Depuis jours il était à la recontre de son Père parti depuis trois jours pour aller à la rencontre de son Père.

Au fond de la salle Astolphe assis prés d'une table lisait à la lueur d'une lampe dans un gros volume qui contenait les titres de la maison d'Adelsberg dont il était proche parent et son oeuïl avide y cherchait quelque droit de pretendre à son heritage. C'était là son étude depuis tengtems constante. Cependant depuis quelques instants un mot échappé à la Comtesse [fol. 5v] l'avait distraît de en secture et desirant en entendre davantage, il fait semblant de se retirer et resta sans etre apercu. [phrase illisible biffée]

Comment mon Ida peut être triste, rappela la Comtesse. Ne sait-elle pas que peu de jours aprés l'arrivée du Comte cette courone ornera son front. Les noces sont publiées, les tournois se préparent, les habits, les bijoux sont prêts. Les jeunes compagnes, mon Ida, tressent deja leurs guirlandes et ton Ernest ivre d'amour et du bonheur ne desire, ne rêve que son amie. Mais que voisje! des larmes coulent dans tes yeux. C'est en vain que tu les détournes, que veut dire cette rougerie et cette pâleur subite [fol. 6r] Ida! ... aurais-tu des secrets que j'ignore? Cacherais-tu quelque chose à ta mère adoptive, à ta meilleure amie ! ... O ! Ma Mère ! dit Ida en se mettant à genoux devant elle et se cachant le visage dans son sein. Votre ta bonté me tue. Ne me parle pas ainsi. J'aime mieux mourir que de t'afliger, ne me demande rien, je t'en prie.

Non mon Ida, je te demande tout reprit la Comtesse en la tenant contre son coeur. Je ne desire que ton bonheur. Dussais-je en etre afligé, dut Ernest en etre malheureux, je remplirai toujours l'engagement sacré que je pris de t'aimer comme une fille et de veiller à ton bonheur, remplis aussi le tien mon Ida et souviens-tu qu'une entiere confiance est la seule recompense qui [fol. 6v] puisse satisfaire mon amour. Mère! tu me gronderas ou bien tu pleureras, dit Ida à demi voix. Tu es bien enfant mon Ida, repondit la Comtesse. Je ne veux ni pleurer ni gronder, je t'en assure. Je veux lire dans ton coeur et t'aider à trouver le bonheur, lève-toi et parle sans crainte.

Non ma Mere repondit Ida, puisque tu veux que je te parle, je le ferai mais à genoux devant toi que j'offense, appuyée sur ton coeur qui me rassure et sans regarder ton visage emprinte de toutes les vertus. Après quelque'hésitation, quelques frases commencées et interrompues, Ida reprit ainsi : Mère! J'aime ton fils de tout mon coeur mais comme un frère et j'aime ton frère comme [fol. $7 \mathrm{r}]$ un époux. À ces mots sa tête tomba sur les genoux de la Comtesse resta quelque tems immobile et sans poir pronor que paisie et tremblée resta quelque tems immobile et sans pouvoir 
De l'amour perdu vers les « Amours » retrouvés. A propos des écrits privés et littéraires de la comtesse Alexandrine Schouvaloff

produire une parole. Les larmes coulèrent enfin de ses yeux. O! Ida dit-elle quel cruel secrêt vins-tu de me découvrir mais achêve ma fille et tu crains rien. Mon coeur affligé saura te plaindre et te consoler. Ida confuse mais rassurée par ces paroles commence ainsi son récit.

Lorsqu'à la mort de mon Pére mon Oncle me recut de ses bras tu sais qu'il lui promis de m'élever comme sa fille et de m'unir à son fils. Destiné à Ernest depuis mon enfance je m'accoutuma à le regarder [fol. $7 \mathrm{v}$ ] comme le compagnon de ma vie entière et je desirai lui plaire et l'aimer. J'endais J'étais heureuse et j'entendais l'autre, notre beauté, nos sentiments, nos naîfs ames et nos richesses. Je trouvais tout cela bien beau et la seule chose qui me semblait un peu injuste dans cette union était la réunion de deux riches héritages dont chacun pouvait rendre un ménage heureux. Je vis ton frère, je pensai qu'il était pauvre et que je voudrais bien lui donner la moitié de mon bien. Il était brave si vaillant, si aimable. Ernest si enfant, Conrad me parlait regardait d'un air si triste, si pénêtré! ... Il partit pour la Palestine et je résolus que je l'aimais plus que ma vie.

Elle achevait à premiers mots que [fol. 8r] les [mots illisible] rebondirent du fond du corps. Les hostes se lèvent, des flambeaux paraîssent dans la cour. La comtesse y vole et se heurte dans les bras de son époux. Ernest serre Ida dans ses bras, au même instant elle appercoit Conrad mais il est revêtu du manteau des Templiers et Ida tomba presque sans sentiment dans les bras du Comte qui l'embrasse. La Comtesse a tout vu, tout compris. Elle remet Ida à la nourice, entraîna le Comte et Conrad et ordonna à Ernest de la suivre.

Le jour commencait à paraître. Après les élans de la joie et de l'amour on se rendit à la Chapelle ou la Sainte Messe fut célébrée avec pompe. Ida que la Comtesse avait été du retenir y assista en pleurant, mais sous long voile cachait ses larmes, à peine osait-elle jetter de tems en tems un regard furtif sur Conrad. Elle ne montra qu'une fois le sien, mais elle crut [fol. 8v] y lire que le désespoir seul avait dicté ses voeux. Elle ne resentit pas le courage d'assister au dejeuner et à se prétendant la fatigue qui l'accablait elle se retira dans sa chambre, se jetta sur son lit et demanda qu'on la laissa seule. Le Comte fut surpris de son absence mais entièrement livré à la joie de ressérer l'aimable et belle Comtesse il accepta l'excuse qu'elle lui donna. Sauf Ernest qui depuis trois jours ne rêvait qu'au bonheur de revoir son amie il y était consterné de l'embarras et de la froideur qu'il avait cru remarquer dans ses regards. Ses yeux interogeraient ceux des autres convives mais que pouvait-il y trouver de consolant. Sa mère au millieu de sa joie le regardait avec tristesse. Le Comte ne pensait pas à lui dans ces moments, [fol. 9r] Conrad pâle et defait tenait ses yeux baissés ou les tournait d'un air distrait sur les tableaux qui décoraient la salle et Astolphe semblait tantôt oecupé d'un joie mensif et tantôt plein d'énergie dont l'expansion avait quelque chose de malin et de decevante. Le pauvre Ernest n'osant interroger personne fut contraint de se retirer comme les autres et de remettre à un autre moment l'explication d'un mistère qu'il ne pouvait comprendre.

Il y songea pendant toute la matinée car le tourment n'aprocha pas de ses yeux. Conrad qui ne n'avait pu s'y livrer davantage monta dans les crénaux espérant que la fraîcheur du matin remettrait le calme dans son sang. Mais tandis que trois âmes amicales se livrent à leur mélancholie, tandis que deux époux s'entretiennent [fol. 9v] du bonheur de se revoir, le crime ouvrit sa trame perfide et calcule de sang froid jusqu'où il peut s'avancer sans se compromettre. Astolphe est retiré dans son appartement. Rup il passait à pas lents lles bras croisés il marche lentement les bras croisés et rêve à ses projets de vengeance. Ruperd debout près de la porte n'ose interrompre ses méditations. Tout à coup Astolphe s'arrête. Un éclair de joie brille sur son front. Le l'at trouvé, dit-il [...] Oui, je l'ai trouvé. Ruperd, ferme les portes [...] les veroü̈lles et écoute-moi. La vengeance malheur plêne déjà sur la tête du Comte et bientôt le deü̈l regnera dans le chateau mes - Ruces ... Ruperd dit Il fait signe à Ruperd de femer rompre le verrouil de la porte et de le suivre dans l'ombragure d'une étroite croisée. [fol. 10r] Tous deux restent un moment sans parler. Astolphe fait sur Ruperd un regard servitateur et presque suspendu. Il rompt enfin le silence. Ruperd! Cette nuit m'a decouvert un secrêt qui change entierement mes projets et assure ma vengeance. J'ai scu .Pourquoi pâlis-tu et un second regard sembla chercher au fond du coeur de l'Ecuyer s'il pouvait lui en dire davantage. As-tu vu Herda; oui Seigneur et voici un philtre dont sept gouttes peuvent rendre la Demoiselle d'Adelsberg entièrement éprise de vous. --- Donne! et Astolphe recommencea \$a marche à marcher lentement. Il revient ensuite à Ruperd. Écoute! luis je compter sur toi J'exige en serment solennel. Je vais te découvrir tous mes secrets. Tremble d'en devoiler la moindre circonstance. Une récompense brillante si je réussis, un cachet si tu parles savant ton partage [fol. 10v] Seigneur je me soumets à tout. Je le crois dit Astolphe en lui jettant un coup d'oeïl atroce. Je 
De l'amour perdu vers les « Amours » retrouvés. A propos des écrits privés et littéraires de la comtesse Alexandrine Schouvaloff

veux le croire entends-tu? J'ai besoin de toi. Je compte sur ta fidélité Ton interret me reprend de toi, mais puisque je ne craigne pas tes scrupules, je veux bien te faire connaître la raison de ma haine contre le Comte et sa famille tu vas fremir. Mes ayeux étaient les Seigneurs Reignants d'Adelsberg. L'un d'eux fatigué du joug Tyrannique de [espace vide] de [espace vide] se révolta contre lui. Il fut vaincu, bani et ses biens passèrent à la branche cadette de la famille. Le comte est l'heritier de cette branche, son Père fit rapeller le mien, lui fit don d'une petite terre et le reçut dans [fol. 11r] son chateau. Je suis né dans ses murs. Instruit par un vieux domestique de l'histoire de ma famille je sentis profondément l'état d'humilation où je me trouvais reduit en étant le protégé de celui dont je devais etre le maître. En vain le Comte me prodigua-t-il les marques d'une accuité outra grande, mon coeur fermé à tout autre sentiment n'éprouvait que celui de la hâ̂ne. Sans cependant la dissimuler, je m'indignais quelquefois de cette bassesse, mais le faible ne doit-il pas avoir ses armes comme le fort? J'avais vingt cinq ans lorsque je vis Isabelle de Souzau de baron du Zelietrack. Je ne sais ce qui me plut sur elle. Son cuir, son caractère étaient opposés au mien. Sans doutte ce contraste m'emflama, il me parut que sa douceur calmerait mon âme [fol. 11v] brûlante. Elle était pauvre et son oncle supposant que des fidés commis ne pouvait lui donner qu'une dot très modique. Ce manque de fortune était un grand obstacle pour moi et je rêvais nuit et jour au moyen de la faire disparaitre lorsque le Comte d'Adalsberg avait me déclarer que son Père avait demandé et obtenu pour lui la main d'Isabelle. Hors d'être de me contenir je me jettais sur lui avec rage, il tira son épée pour se deffendre, nous nous battimes et je le blessai griévement. Le vieux comte indigné fut donné aux prieres de son fils. Il me fit saisir par sa garde et renfermer dans une celule du couvent de Saint Siegfrid. Je passais cinq ans dans cette étroite forteresse. [fol. 12r] Le jeune Comte Léopold m'écrivait souvent et m'envoyait [...] ce qui pouvait m'etre agréable ou nécessaire. Ses lettres respiraient en apparance la plus fade amitié et les plus vifs regrêts de ne pouvoir toucher son Père. Je ne repondis pas d'abord mais le tems et la réflexion n'ayant nuisi l'esprit et fait enfauter des projets de vengeance, j'eus l'air de me prendre à ses priéres et je lui demandai même d'oublier mon troublant de jeunesse et mon amour insensé dont il n'anéantait plus la moindre trace dans mon coeur. Rien n'était moins vrai. Ma passion devait s'être changée en fureur et l'espoir d'anéantir le rival heureux qui depuis des années jouissait des charmes d'Isabelle était le seul qui me fut cher. Lorsque je songais à son mariage, à la naissance de son [fol. 12v] fils, aux richesses dont il était entourées, je secouais avec rage les bareaux de fer de ma prison et j'appellais à grand cris le Demon de l'Enfer à mon secours. Les moines effrayés me crurent en demence. Je compris qu'ils voulaient rendre compte au chateau de mes acces de fureur. Je promis d'être plus tranquile et je le devins en apparance. Le vieux Comte mourut en me pardonant et Léopold vint me retirer de ma captivité. Il eut l'air touché jusqu'aux larmes de ma maigresse et de ma souffrance. Il se jetta dans mes bras et me conduisit dans ce chateau. Craignant de me trahir, je pretai un morne silence qui devient mon caractère habituel. Sourde et retireé dans mon appartement [fol. 13v] Je laisse croire à tout le monde qu'une mélancholie profonde suite de ma captivité ne me fait tolérer que la retracte et le repos. J'observais la Comtesse et je vis que rien n'aurait pu la séduire, son fol amour pour son époux finit par exciter tous mes mépris et je la compris des lors parmi les objets qui méritaient ma haine. Mon plan était arrêté depuis longtems je prétentai mon pélerinage et c'est alors, continua Astolphe à voix basse en serrant le bras de Ruperd, c'est alors que je me fis recevoir dans le tribunal terrible dont je suis membre et dont toi même es un affiché. C'est aux francs juges que je demanderai justice, c'est devant eux qu'il faut entrainer Ernest et accuser Conrad [fol. 13r] Conrad, Seigneur, demanda Rupert étonné. Dès longtems, reprit Astolphe j'avais surpris l'amour de Conrad pour la jeune Ida. Mais j'ignorais qu'elle y fut sensible. Il est inutile de te dire comment je l'apris. Tu sais mes projets sur elle. Redevenu ce que je suis par droit de naissance, Comte et Seigneur Souzerain d'Adelsberg, je compte en l'épousant réunir à mes biens son riche héritage et c'est sous les yeux d'Isabelle désolée. C'est sur la tombe de son époux et de son fils et de son frère que cet himen sera conclu.

Parles cependant Ruperd, pourquoi gardes tu le silence.

Seigneur, je suis si étourdi de tout ce que vous venés de me dire que je ne sais que vous repondre. » 


\section{Conclusion}

Notre visite sommaire de l'atelier d'écriture de la comtesse Alexandrine Schouwaloff s'est montrée prometteuse. L'œuvre manuscrite de cette aristocrate, déracinée de sa patrie et de son milieu familial, s'inscrit parfaitement dans son temps et épouse ses articulations sur plusieurs plans. Elle ressort de ses origines, de sa vie de femme divorcée et venue d'ailleurs, de ses voyages et de ses lectures cosmopolites, de son désir d'accéder à la foi chrétienne par le sentiment, de mettre en harmonie la religion, la nature, les passions du cœur et la vertu. Ses écrits et ses lectures témoignent le cosmopolitisme littéraire de l'époque préromantique, son écriture poursuit les modèles des femmes écrivains qui s'intéressent à la morale, qui cherchent l'unité de mouvement pour le monde matériel et pour le monde spirituel et rêvent d'une société harmonieuse. Dans certains récits (Mémoires de Romina Grobis), Alexandrine arrive à trouver le ton ironique pour ridiculiser la mauvaise qualité et la surproduction des récits mémorialistes quasi documentaires dans lesquelles on ne propose que des fabulations romancées et n'impose que ses mérites personnels. Elle critique aussi l'influence dangereuse et la manipulation des opinions des lecteurs par la presse.

\section{BIBLIOGRAPHIE}

\section{Sources primaires}

Archives de la famille Dietrichstein à Brno en Moravie : MZA Brno, fond G 140, boîte 583 (généalogie, chapelle privée, distinction, divorce, souvenirs et mémoires) ; boîtes 584-585 (essais littéraires); boîtes 586-587 et 589 (correspondance).

Conversion de S. A. Madame la Princesse Alexandrine de Dietrichstein née Comtesse Schouwaloff racontée par elle-même. D'après le manuscrit de l'auteur (1879), publié par sa petite-fille Alexandrine Mensdorff- Pouilly, Sauton, Paris. MZA Brno, RAD, inv. 2503, cote 1312

\section{Bibliographie sommaire des sources secondaires}

AMBrIÈRE Madeleine (1990), Précis de littérature française du XIX siècle, Paris, Presses Universitaires de France.

CERMAN Ivo (2011), Šlechtická kultura v 18. století. Filozofové, mystici, politici, Praha, Nakladatelství Lidové noviny.

GENETTE Gérard (1991), Fiction et diction, Paris, Éditions du Seuil.

GIFFORD Paul ; SCHMID Marion (eds) (2007), La création en acte. Devenir de la critique génétique, Editions Rodopi B. V. Amsterdam - New York, New York.

HAY Louis, Critique génétique et la théorie littéraire: quelques remarques, in : GIFFORD Paul ; SCHMID Marion (eds) (2007), La création en acte. Devenir de la critique génétique, Editions Rodopi B. V. Amsterdam-New York, New York, p. 13-27. 
KaFANOVA Olga B. (2004), La Réception de l'œuvre de Jean-François Marmontel en Russie, in: Poussou Jean-Pierre (éd.) L'Influence française en Russie au XVIII ${ }^{e}$ siècle, Paris, Institut d'Études Slaves, p. 237-252.

MAŠEK Petr (2008), Šlechtické rody v zemích koruny české od Bílé hory po současnost, I. díl (A- M), Praha, Argo.

MAŠEK Petr (1990), Zámecké knihovny na státním zámku Úsov. Miscellanea odd. rukopisù a vzácných tisků Národní knihovny, 7/2, p. 67-94.

MUSILOVÁ Martina (2011), Le cycle de l'amour écrit par la princesse Alexandrine de Dietrichstein, née comtesse de Schouwaloff : Amour allemand, Amour italien, Amour russe, Amour suisse, mémoire de licence dactylographié, dirigé par Jitka Radimská, Université de Bohême du Sud, České Budějovice, 83 p. http://theses.cz/id/27ilo0/

MusiLOVÁ Martina (2014), «Gâtée par le monde.» Literární dílo kněžny Alexandry z Dietrichsteina, mémoire de maîtrise dactylographié, dirigé par Ivo Cerman, Université de Bohême du Sud, České Budějovice, $147 \mathrm{p}$. http://theses.cz/id/dc9s82/

NiQUeUX Michel (2009), Typologie des récits de conversion au catholicisme (première moitié du XIX ${ }^{\mathrm{e}}$ siècle), in: NIQUEUX Michel (coord.), Journée d'étude Religion et Nation, communications de la journée d'étude organisée par l'Institut européen Est-Ouest (UMR 5206) à l'ENS de Lyon le 8 juin 2009. http://institut-est-ouest.ens-lyon.fr/spip.php?article287\&lang=fr

OBRŠLÍK Jindřich ; ŘEZNíČEK Jan ; VOLDÁN Vladimír (1979), Rodinný archiv Dietrichštejnů (1097) 1222-1944, I-III, Brno, Státní oblastní archiv.

RJEOUTSKI Vladislav (2007), La langue française en Russie au siècle des Lumières: éléments pour une histoire sociale, in: Multilinguisme et multiculturalité dans l'Europe des Lumières (Acte du Séminaire international des jeunes dix-huitiémistes 2004) / Multilinguism and Muticulturalism in Enlightenment Europe (Proceedings of The International Seminar for Young Eihteenth-Century Scholars 2004), études réunies par/ edited by U. HaskinsGonthier et A. Sandrier, Paris, Honoré Champion, p. 101-126.

STROEV Alexandre (2009), Le réseau des connaissances et des correspondants russes du prince Charles-Joseph de Ligne, Nouvelles Annales Prince de Ligne, XVIII, Bruxelles, Groupe d'Etudes lignistes, p. 7-65.

ŠVAŘíčKOVÁ-SLABÁKOVÁ Radmila (2007), Rodinné strategie šlechty. Mensdorffové-Pouilly v 19. století, Praha, Argo.

TOVAČOVSKÝ Jaroslav (1997), Rodové vztahy Valdštejnů, Nosticů a Dietrichsteinů s ruskou šlechtou, Heraldika a genealogie, 3-4, Klub pro českou heraldiku a genealogii, p. 199-228. 


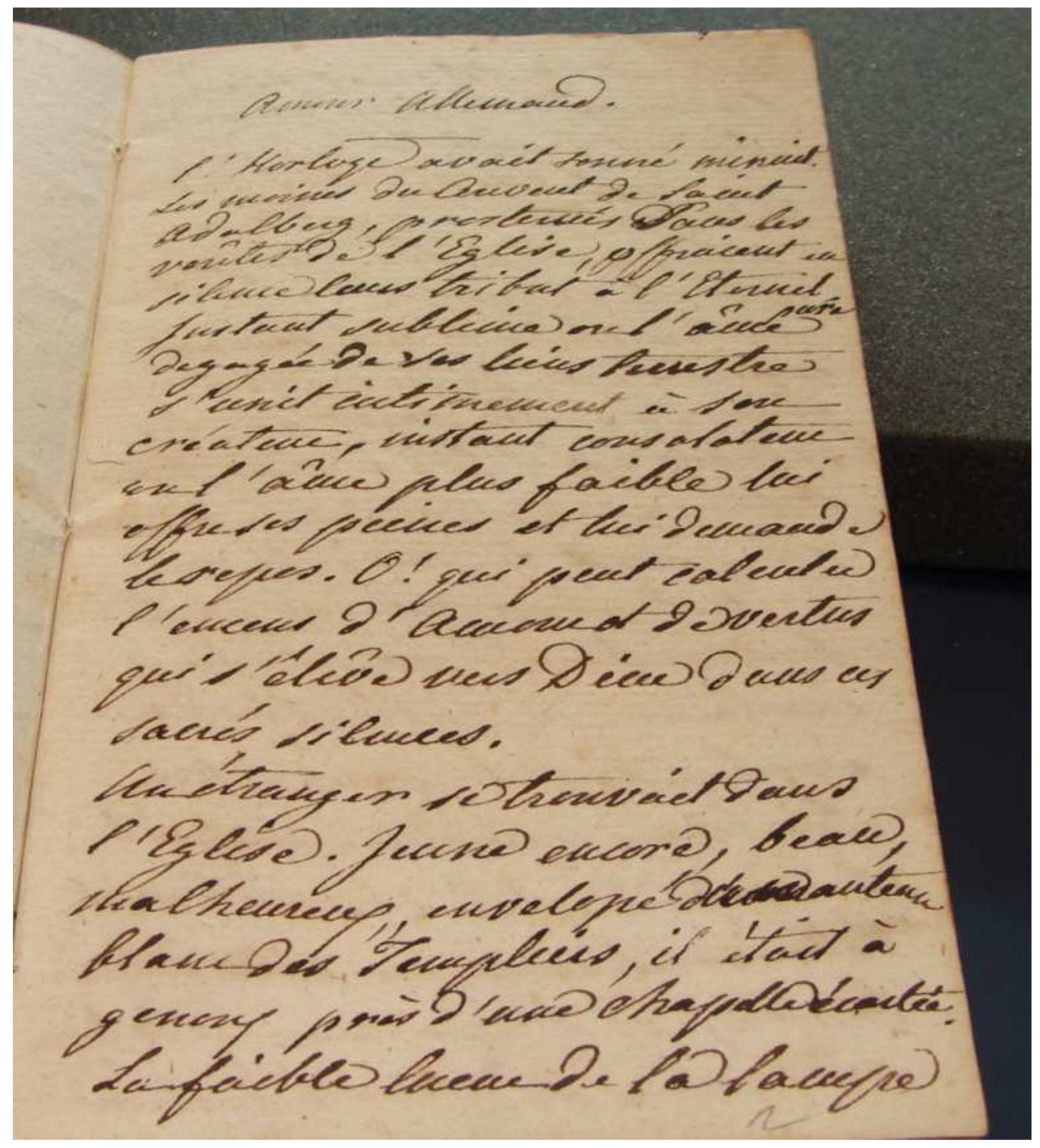

Incipit manuscrit du conte inédit Amour allemand. MZA Brno, G 140, boîte 584, inv. 2455, fol. 2r. Voir p. 114 ci-dessus. 